\title{
Legionella in Clinical Specimens and Hospital Water Supply Facilities: Molecular Detection and Genotyping of the Isolates
}

\author{
J.A. Qasem ${ }^{\text {a }} \quad$ A.S. Mustafa ${ }^{\text {b }} \quad$ Z.U. Khan ${ }^{\text {b }}$ \\ a Department of Applied Medical Sciences, College of Health Sciences, The Public Authority for Applied Education \\ and Training, and ${ }^{\mathrm{b}}$ Department of Microbiology, Faculty of Medicine, Kuwait University, Kuwait
}

\section{Key Words}

Legionella $\cdot$ Polymerase chain reaction $\cdot$ Random amplification of polymorphic DNA · Typing methods

\begin{abstract}
Objective: To evaluate genus- and species-specific polymerase chain reactions (PCRs) for the detection of the genus Legionella and the species Legionella pneumophila in clinical specimens and hospital water supplies, and to establish a simple and reproducible random amplification of polymorphic DNA (RAPD)-PCR technique for genotyping of Legionella. Materials and Methods: A total of 70 respiratory tract specimens (bronchoalveolar lavage: $n=46$; endotracheal secretions: $n=9$; sputum: $n=15$ ) from patients with atypical pneumonia, and 283 environmental samples (water: 20; swabs: 263) collected from water storage and supply facilities of the Mubarak Al-Kabeer Hospital, Kuwait, were tested by culture and genus-specific PCR for the detection of Legionella. The L. pneumophila isolates were subsequently typed by serology and RAPD-PCR using serotype-specific sera and arbitrary primers, respectively. Results: Of the 70 clinical samples, culture yielded 2 (2.9\%) whereas genus-specific PCR detected Legionella in $20(28.6 \%)$ samples. The 2 culture-positive specimens were also positive for L.-pneumophila-specific PCR. Testing of swab and water samples by culture and genus-specific PCR yielded 61 (21.6\%) and 67 (23.7\%)
\end{abstract}

positive samples, respectively. All of the 61 culture-positive samples were also positive by genus-specific PCR and 45 of them were positive for L.-pneumophila-specific PCR. Serological typing of 43 L. pneumophila isolates showed that 8 of these belonged to serotype 1 and 35 to serotype 3; however, RAPD-PCR analyses demonstrated polymorphisms among the isolates of both serotypes. Conclusion: A higher association between PCR and culture was observed for the environmental samples than for the clinical samples. The application of genus- and species-specific PCRs and RAPD is useful in the detection and typing of Legionella in clinical and environmental samples.

Copyright $\odot 2007$ S. Karger AG, Basel

\section{Introduction}

Legionella is a naturally occurring aquatic bacterium. It has been found to inhabit many kinds of natural and man-made water reservoirs [1-3]. Outbreaks of nosocomial legionellosis are a frequent problem in hospital environment [3], and it has been suggested that some sporadic cases of the disease may arise from contaminated water systems of the hospitals [4]. Among the currently available methods for the routine diagnosis of Legionella infection, culture is the most specific method but its sensitivity is variable and unreliable because of the presence

\section{KARGER \\ Fax +4161306 1234 \\ E-Mail karger@karger.ch}

www.karger.com
(C) 2007 S. Karger AG, Basel

1011-7571/08/0171-0049\$24.50/0

Accessible online at:

www.karger.com/mpp
Dr. Jafar A. Qasem

College of Health Sciences, Department of Applied Medical Sciences

The Public Authority for Applied Education and Training

PO Box 9508, Ahmadi 61006 (Kuwait)

Tel. +965 481 2710, ext. 6713, Fax +965 3906099 , E-Mail qasemj@paaet.edu.kw 
of a low number of viable organisms in most clinical specimens [5-7]. Serology for antibody detection is not of value in the acute phase of the disease and serological methods have not been satisfactorily validated in the diagnosis of non-Legionella-pneumophila infections [5-7]. Immunoflourescence assay with respiratory tract specimens is specific but insensitive [5]. The detection of Legionella antigen in urine using enzyme immunoassay or radioimmunoassay is very specific but the commercially available systems only detect L. pneumophila infection [5-7]. Therefore, there is a need to establish and validate other rapid and specific methods that are more sensitive than the currently available methods of culture and serology. In the past, polymerase chain reaction (PCR) assays targeting genus- and species-specific DNA have been established for early and sensitive detection of Legionella in clinical specimens [8-10]. In this study, we have evaluated genus- and species-specific PCRs targeting $5 S$ rRNA and mip genes for the detection of the genus Legionella and the species L. pneumophila in respiratory tract specimens from patients with atypical pneumonia and environmental water samples.

In addition to sensitive detection, typing Legionella strains is of considerable importance in ascertaining sources of community outbreaks or nosocomially acquired legionellosis [11]. Therefore, highly discriminatory typing methods are required to provide useful information regarding common-source cases and the identification of sources of infection. Serological typing is often used but it is insufficiently discriminatory when a given serogroup comprises only a few antigenically distinct subtypes, as for L. pneumophila serogroup 6 [12]. Therefore, discriminatory molecular typing methods should be applied to clinical and presumptively linked environmental strains in order to detect the source of infection. In this regard, random amplification of polymorphic DNA (RAPD)-PCR has been introduced as a reliable method to distinguish between phenotypically/serologically similar bacterial species and strains including legionellae $[1,2]$. In this study, RAPD-PCR was used for genotypic analysis of Legionella isolates from clinical and environmental sources.

\section{Materials and Methods}

\section{Specimen Collection}

Seventy clinical specimens comprising bronchoalveolar lavage (BAL; $n=46$ ), endotracheal secretions (ETS; $n=9$ ) and sputum $(n=15)$ were collected from atypical pneumonia patients admitted to the Mubarak Al-Kabeer Hospital, Kuwait. Two hun-
Table 1. List of standard L. pneumophila and non-L.-pneumophila species and strains used in this study

\begin{tabular}{lll}
\hline Legionella species & Strains \\
\hline $1-10$ & L. pneumophila & NCTC $(11192,11230,11232,11233$, \\
& & $11405,11406,11984,11985,11986$, \\
& & $12000)$ \\
$11-12$ & L. pneumophila & ATCC $(33152,33159)$ \\
13 & L. bozemanii & NCTC 11368 \\
14 & L. dumoffii & NCTC 11370 \\
15 & L. micdadei & NCTC 11371 \\
16 & L. gormanii & NCTC 11401 \\
17 & L. longbeachae & NCTC 11477 \\
\hline
\end{tabular}

dred and eighty-three environmental samples comprising 263 swabs from faucets of washbasins and showerheads and 20 water samples from the water storage facilities of the Mubarak-Al-Kabeer Hospital, Kuwait, were collected. The swab samples were taken by twirling well inside the taps of washbasins and showerheads.

\section{Legionella Strains, Culture and DNA Isolation}

Reference strains of Legionella $(\mathrm{n}=17)$ were obtained from the National Collection of Type Cultures (NCTC) and American Type Culture Collection (ATCC) (table 1). The reference strains, clinical samples and swabs were cultured on buffered charcoal yeast extract agar (BCYEA- $\alpha$ ) supplemented with L-cysteine, ferric pyrophosphate and antibiotics according to standard procedures [13]. From the water samples (2.5 liters each), $100 \mu$ l were used for culture on BCYEA- $\alpha$ with antibiotics and supplements. The remaining water was filtered through a millipore filtration assembly $(0.2 \mu \mathrm{m}$, Nalgene filter). The membrane filter was taken out aseptically and cut into small pieces $\left(1-2 \mathrm{~cm}^{2}\right)$. One piece was used for culture on BCYEA- $\alpha$ plates with antibiotics and supplements. All the culture plates were incubated at $37^{\circ} \mathrm{C}$ with $5 \% \mathrm{CO}_{2}$ and observed up to 14 days for growth of Legionella. The rest of the membrane filter pieces along with $400 \mu$ l filtered water were used for DNA extraction by vortexing thoroughly and boiled in an Eppendorf tube for $10 \mathrm{~min}$. After boiling, the tubes were kept on ice for $5 \mathrm{~min}$, centrifuged at 14,000 rpm for $10 \mathrm{~min}$ and $10 \mu \mathrm{l}$ of the supernatant was used for PCR. DNA from clinical samples was isolated by an alkaline lysis method followed by phenol/chloroform extraction using standard procedures [14]. DNA from standard strains and culture isolates was extracted by a heat lysis method [15].

\section{PCR Amplification Analysis}

Two sets of oligonucleotide primers capable of detecting organisms of the genus Legionella and the species L. pneumophila [8] were obtained from Genosys Biotechnologies, UK. The sequence of primers, the target gene and the expected size of amplified DNA are given in table 2.

Amplification of target DNA was carried out in thermal cyclers (GeneAmp PCR systems 9600 and 2400, Perkin-Elmer Cetus Instruments, Norwalk, Conn., USA). All DNA amplifications 
Table 2. The sequence of PCR primers, the target gene and the expected size of amplified DNA

\begin{tabular}{llll}
\hline Primer & Sequence & Target gene & Size of amplified DNA \\
\hline L5SL9 & 5'-ACTATAGCGATTTGGAACCA-3' & 5S rRNA & 104 bp \\
L5SR93 & 5'-GCGATGACCTACTTTCGCAT-3' & 5S rRNA & 104 bp \\
LmipL920 & 5'-GCTACAGACAAGGATAAGTTG-3' & mip & $650 \mathrm{bp}$ \\
LmipR1548 & 5'-GTTTTGTATGACTTTAATTCA-3' & mip & $650 \mathrm{bp}$ \\
\hline
\end{tabular}

including the appropriate positive and negative controls were performed as described previously [16]. The PCR products were resolved by agarose gel electrophoresis and photographed under UV light using standard procedures [17]. The samples showing DNA bands of expected size were scored positive.

L. pneumophila isolates were serotyped by using a serotyping kit (Seiken, Denka Co. Ltd., Chuo-ku, Tokyo, Japan) according to the manufacturer's recommendations.

\section{Molecular Typing of Legionella by RAPD-PCR}

RAPD-PCR was standardized with 7 different decamer oligonucleotide primers (OPB-01, OPJ-05, OPJ-06, OPJ-07, OPJ-11, OPN-02, OPJ-15) $[18,19]$ with DNA from 17 reference strains of Legionella. All the primers were purchased from Operon Inc., Alameda, Calif., USA. Amplification reactions were performed in $25 \mu \mathrm{l}$ reaction mixture using GeneAmp PCR System 2400 (Perkin-Elmer Cetus Instruments). The thermal cycler was programmed with a denaturing precycle of $5 \mathrm{~min}$ at $95^{\circ} \mathrm{C}$, followed by 45 cycles of $1 \mathrm{~min}$ at $95^{\circ} \mathrm{C}, 1 \mathrm{~min}$ at $36^{\circ} \mathrm{C}$, and $2 \mathrm{~min}$ at $72^{\circ} \mathrm{C}$. Each reaction mixture contained $200 \mu \mathrm{M}$ of each dNTP, $1.2 \mu \mathrm{M}$ primer, 50 ng template DNA and 1.5 U Ultima Taq DNA polymerase (Perkin Elmer, USA) in $1 \times$ PCR buffer $(10 \mathrm{mM}$ Tris $\mathrm{HCl}$, $\mathrm{pH}$ 8.0, $50 \mathrm{~mm} \mathrm{KCl}, 1.5 \mathrm{~mm} \mathrm{MgCl}_{2}, 0.1 \%$ Triton X-100). The RAPD products were resolved by agarose gel electrophoresis and photographed under UV light [17]. The computer analysis of RAPD patterns was performed by using GelWork 1D Advance version 4.01 (No-Linear Dynamics, UVP Inc., Upland, Calif., USA). The resulting matrix of pairwise distance was used to construct a dendrogram employing the unweighted pair-group method with arithmetic mean included in the molecular evolutionary genetics analysis software [20]. All pictures were digitized with UVP video and analyzed with the computer software Grabit, Annotating Graphic Grabber (32 bit) version 2.59 (UVP Inc.).

\section{Results}

Detection of Legionella in Clinical and Environmental Samples by Culture and PCR

Of the 70 respiratory tract specimens (BAL: $n=46$; ETS: $\mathrm{n}=9$; sputum: $\mathrm{n}=15$ ) only 2 specimens (BAL) were positive by culture, whereas 20 specimens (BAL: $n=13$; ETS: $n=3$; sputum: $n=4$ ) were positive by the genusspecific PCR (table 3 ). The 2 culture-positive specimens
Table 3. Comparison of culture with genus- and species-specific PCR for the detection of Legionella in clinical and environmental samples

\begin{tabular}{|c|c|c|c|c|}
\hline \multirow[t]{2}{*}{ Sample } & \multirow[t]{2}{*}{ Total } & \multirow{2}{*}{$\begin{array}{l}\text { Culture } \\
\text { positive }\end{array}$} & \multicolumn{2}{|c|}{ PCR positive for } \\
\hline & & & Legionella & L. pneumophila \\
\hline \multicolumn{5}{|c|}{ Clinical specimens } \\
\hline BAL & 46 & 2 & 13 & 2 \\
\hline ETS & 9 & 0 & 3 & 0 \\
\hline Sputum & 15 & 0 & 4 & 0 \\
\hline Total & 70 & 2 & 20 & 2 \\
\hline \multicolumn{5}{|c|}{ Environmental samples } \\
\hline Water & 20 & 0 & 6 & 2 \\
\hline Swabs & 263 & 61 & 61 & 43 \\
\hline Total & 283 & 61 & 67 & 45 \\
\hline
\end{tabular}

were also L. pneumophila positive by species-specific PCR. With respect to environmental specimens, 61 of 263 swab samples were Legionella positive by culture as well as genus-specific PCR. Of the 20 water samples tested, only genus-specific PCR yielded positive results with 6 samples (table 3 ). The results of species-specific PCR showed that 2 of the 6 water and 43 of the $61 \mathrm{swab}$ samples positive for genus-specific PCR had L. pneumophila, while the remaining 22 were other kinds of Legionella.

\section{Molecular Typing of Legionella by RAPD-PCR}

Of the 7 primers tested, OPN-02 was found best because it yielded a stable and reproducible number of bands with maximum differentiation between different standard strains of Legionella (fig. 1; results given for OPN-02). RAPD analysis showed that L. pneumophila strains ATCC 33152 and ATCC 33159 were the most similar in their band patterns, whereas L. pneumophila NCTC 11192 gave the most varied band pattern. All of the non-L.-pneumophila strains, i.e. Legionella bozemanii NCTC 11368, Legionella dumoffii NCTC 11370, Legionel- 


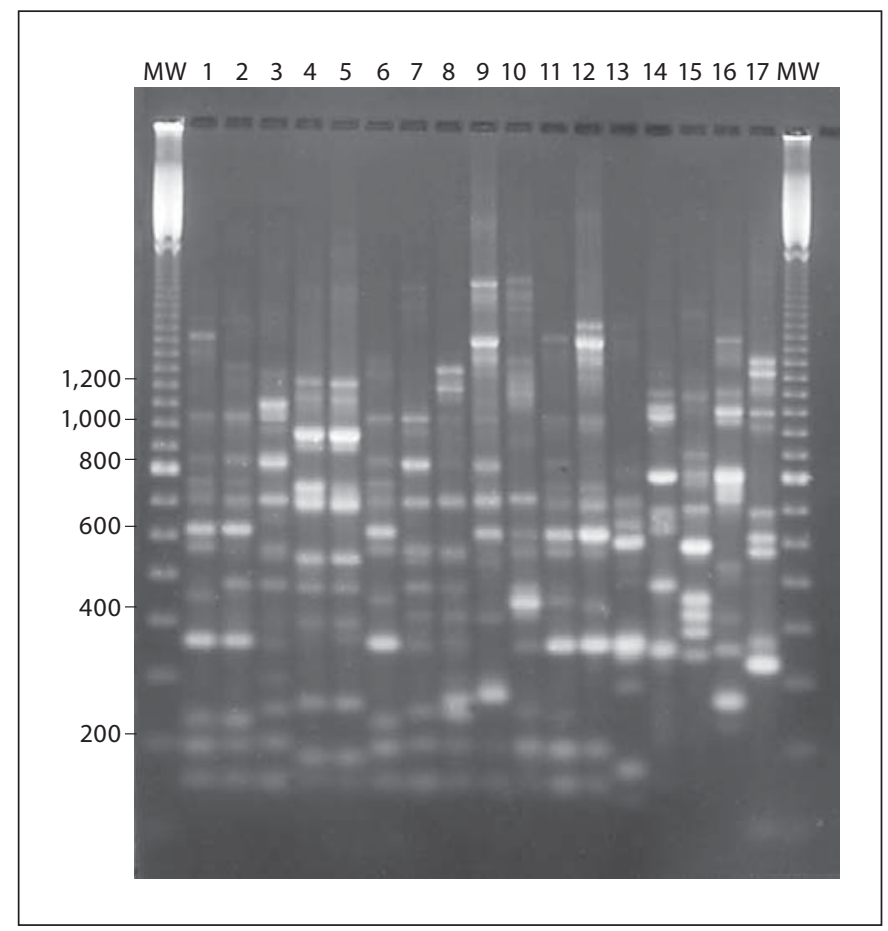

Fig. 1. RAPD fingerprints of Legionella reference strains using primer OPN-02. Lane MW = Molecular size marker (100 bp DNA ladder); lanes 1-10 = L. pneumophila strains NCTC 11192, 11230, 11232, 11233, 11405, 11406, 11984, 11985, 11986 and 12000; lanes 11-12 = L. pneumophila strains ATCC 33152 and 33159; lanes 13-17 = non-L.-pneumophila strains L. bozemanii NCTC 11368, L. dumoffii NCTC 11370, L. micdadei NCTC 11371, L. gormanii NCTC 11401 and L. longbeachae NCTC 11477.

la micdadei NCTC 11371, Legionella gormanii NCTC 11401, Legionella longbeachae NCTC 11477, gave very different band patterns (fig. 1). The RAPD-PCR analysis was extended to include 17 isolates of legionellae other than L. pneumophila and 43 L. pneumophila isolates obtained from the swab samples. All of the isolates of legionellae other than L. pneumophila showed similar DNA band patterns with RAPD-PCR (fig. 2). Serogrouping of the 43 L. pneumophila isolates from environmental swabs with standard antisera showed that 8 and 35 isolates belonged to serogroup 1 and serogroup 3, respectively. However, RAPD-PCR analyses demonstrated that the isolates of both serogroups could further be subdivided into clusters, with 2 major clusters (A and B) in serotype 1 (fig. 3a), and 4 major clusters (A, B, C and D) in serotype 3 isolates (fig. $3 b$ ).

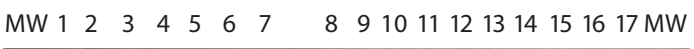

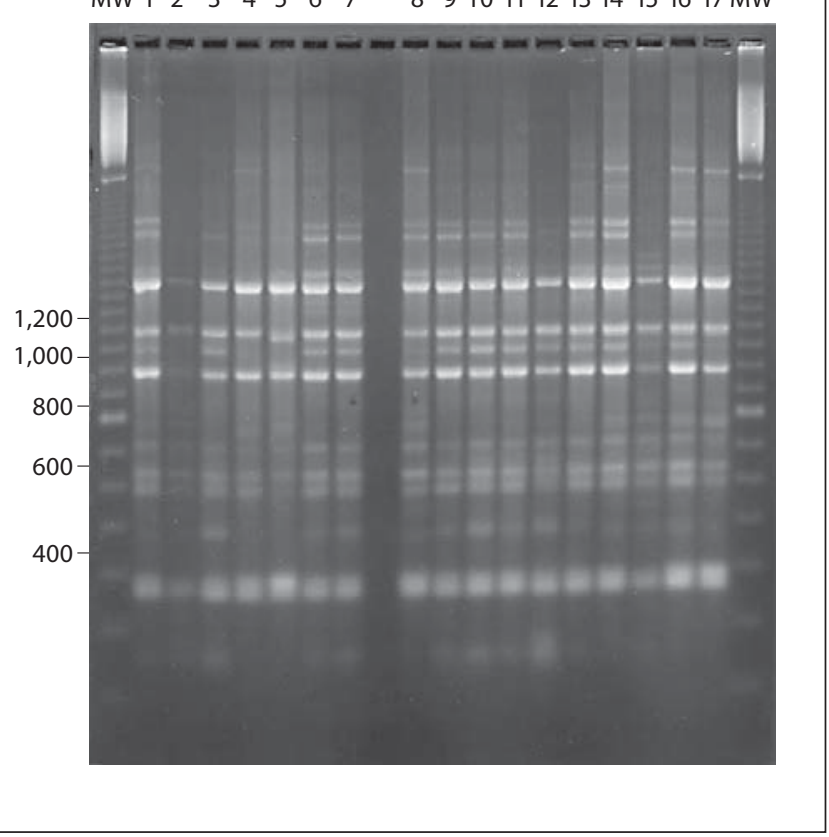

Fig. 2. RAPD band patterns of non-L.-pneumophila isolates with primer OPN-02. Lanes MW are molecular size markers (100 bp DNA ladder); lanes 1-17 are environmental isolates of Legionella.

\section{Discussion}

The genus-specific and species-specific primers used in this study targeted a 104-bp DNA segment from the 5S RNA gene and a 650-bp DNA fragment from the mip gene specific for L. pneumophila, respectively [8]. Testing of 17 reference species and strains of Legionella confirmed the genus and species specificity of the primers of this study (data not shown). The low rate of isolation of Legionella species from clinical specimens by culture $(2.9 \%)$ is consistent with previous findings [4]. For the detection of $L e$ gionella in clinical specimens, PCR (28.6\%) was more sensitive than culture $(2.9 \%)$ but comparatively the same in environmental samples using PCR (21.6\%) and culture $(23.7 \%)$, respectively. This finding may reflect the fact that patients' defense system could lyse bacterial cells releasing bacterial DNA in the blood/tissue, which is detected by PCR, thereby leading to positive PCR and negative culture. Equally important is the fact that at the early stages of infection the number of cells is too low to be detected by culture. The other possible explanation for high positivity of PCR could be contamination of DNA, generally recognized as limitation of PCR [21]. 
Furthermore, the results showed that none of the water samples grew Legionella by culture but $6(30 \%)$ of these yielded positive results by genus-specific PCR, 2 of which were proved to be L. pneumophila by species-specific PCR (table 3). In comparison to the results from the water samples, swabs collected from overhead showers and washbasin faucets (which had considerable rusty deposits) yielded much higher culture and PCR positivity (table 3), suggesting that microniches in the water supply system can serve as a constant source of legionellae, and may also shield these organisms from the adverse effect of chlorination. This scenario appears to be somewhat similar to catheter-associated bacteremia where biofilm formation protects the pathogen from the action of antimicrobial agents [22].

Interestingly, 22 (29.5\%) of the 67 isolates from the environmental source appeared to be legionellae other than L. pneumophila by species-specific PCR. This observation is in accordance with earlier reports [23, 24]. However, some other investigators have reported only L. pneumophila from the water sources $[25,26]$. The precise reasons for these differences in the occurrence of legionellae in water supply systems are not known, but could be attributed to the type of isolation medium used or to the preference of different Legionella species for a particular water habitat. Using BCYE medium, Ta et al. [27] were able to isolate only non-L.-pneumophila species from water samples and noted that these organisms grew poorly on all the selective media. Our results confirm the previous studies that PCR is more sensitive than culture and serology to detect legionellae in clinical and environmental samples $[4,7]$. One of the problems with culture is overgrowth by contaminating organisms and PCR can overcome this problem and thereby improves the sensitivity of detection [28]. In addition, PCR was able to identify L. pneumophila in culture-negative specimens, the most common Legionella species involved in human infections. Thus, PCR could be a highly valuable tool for assisting clinicians in the rapid diagnosis of atypical pneumonia caused by Legionella spp.

In addition to establishing a sensitive molecular technique for the detection of Legionella in clinical and environmental samples, we also established a simple and reproducible RAPD technique for genotyping Legionella. RAPD analysis has been used mostly for intraspecies discrimination in epidemiological studies $[1,2]$. Our results showed that RAPD analysis is highly suited to differentiate between related and unrelated Legionella isolates and strains. The unrelated isolates and strains showed highly variable RAPD patterns, whereas the related isolates and
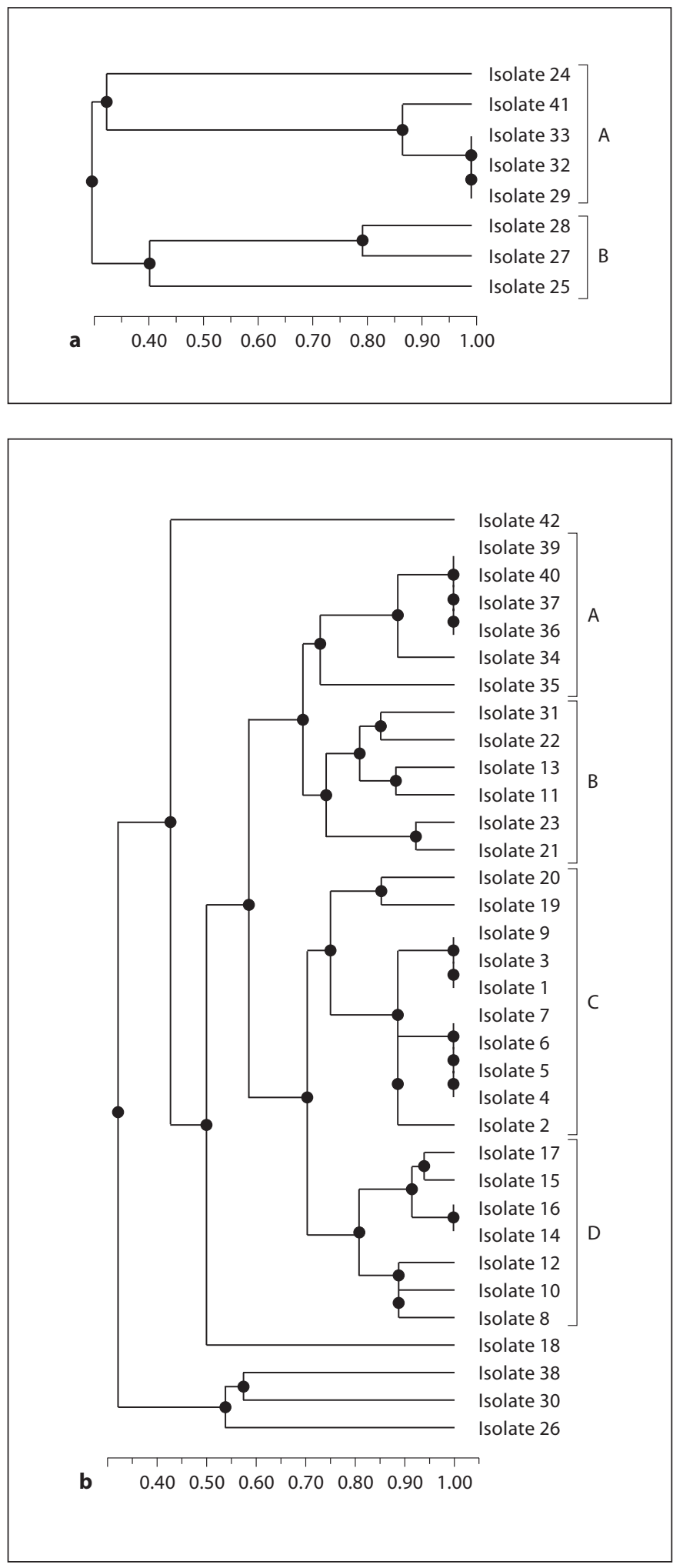

Fig. 3. Dendrogram generated from the RAPD profiles of the 43 L. pneumophila isolates. a L. pneumophila serogroup 1 isolates. b L. pneumophila serogroup 3 isolates. The ruler indicates the similarity percentages for the different dendrograms generated. 
strains showed either identical or less variable RAPD patterns. Furthermore, the results of this study showed a high degree of genetic diversity among the different reference strains of Legionella. These results suggest that RAPD analysis could reliably be used for genotypic analysis and for tracing the source of infection in a hospital environment. It further showed that as compared to the serotyping method currently used to type L. pneumophila strains and isolates, the RAPD analysis is a more powerful typing technique as it could subdivide strains of serogroup 1 and serogroup 3. Thus, RAPD analysis is a better tool to discriminate between isolates as compared to serotyping, and therefore, it could be more informative than serotyping in epidemiological studies and in tracking the origin of a possible hospital infection. The high frequency of $L e-$ gionella isolation from hospital water supply facilities in our study suggests that aerosols generated in bathrooms and washbasins could be a potent source of nosocomial legionellosis. Therefore, monitoring of hospital water supply for Legionella should be helpful in enforcing adequate containment measures [29] and minimizing the risk of hospital-associated Legionella infections.

\section{Conclusion}

The study demonstrates the usefulness of PCR for sensitive detection and identification of organisms belonging to the genus Legionella and the species L. pneumophila as a higher rate of positivity was detected in clinical samples by PCR than by culture. However, similar percentages were identified in environmental samples by both techniques. In addition, this study has shown that RAPD is a simple and reliable molecular method for genotyping of legionellae from clinical as well as environmental sources, which could be important in tracking the origin of a possible hospital infection.

\section{Acknowledgments}

This study was supported by the Kuwait Foundation for Advancement of Sciences grant 96-07-06. The authors are thankful for the cooperation received from the infection control staff of Mubarak Al-Kabeer Hospital and the technical help provided by Ms. S. George.

\section{References}

1 Costa J, Tiago I, da Costa MS, Verissimo A: Presence and persistence of Legionella spp in groundwater. Appl Environ Microbiol 2005; 71:663-671.

2 Perola O, Kauppinen J, Kusnetsov J, Karkkainen UM, Luck PC, Katila ML: Persistent Legionella pneumophila colonization of a hospital water supply: efficacy of control methods and a molecular epidemiological analysis. APMIS 2005;113:45-53.

-3 Stout JE, Yu VL: Hospital-acquired Legionnaires' disease: new developments. Curr Opin Infect Dis 2003;16:337-341.

- 4 O’Neill E, Humphreys H: Surveillance of hospital water and primary prevention of nosocomial legionellosis: what is the evidence? J Hosp Infect 2005;59:273-279.

5 Hindiyeh M, Carroll KC: Laboratory diagnosis of atypical pneumonia. Semin Respir Infect 2000;15:101-113.

-6 Waterer GW, Baselski VS, Wunderink RG: Legionella and community-acquired pneumonia: a review of current diagnostic tests from a clinician's viewpoint. Am J Med 2001; 110:41-48.

7 Den Boer JW, Yzerman EP: Diagnosis of Legionella infection in Legionnaires' disease. Eur J Clin Microbiol Infect Dis 2004;23:871878 .
8 Mahbubani MH, Bej AK, Miller R, Haff L, DiCesare J, Atlas RM: Detection of Legionella with polymerase chain reaction and gene probe methods. Mol Cell Probes 1990;4:175187.

-9 Murdoch DR, Walford EJ, Jennings LC Light GJ, Schousboe MI, Chereshsky AY, Chambers ST, Town GI: Use of the polymerase chain reaction to detect Legionella DNA in urine and serum samples from patients with pneumonia. Clin Infect Dis 1996;23:475480.

10 Matsiota-Bernard P, Waser S, Vrioni G: Detection of Legionella pneumophila DNA in urine and serum samples from patients with pneumonia. Clin Microbiol Infect 2000;6: 223-225.

11 Joly JR, Winn WC: Correlation of subtypes of Legionella pneumophila defined by monoclonal antibodies with epidemiological classification of cases and environmental sources. J Infect Dis 1984;150:667-671.

12 McKinney RM, Kuffner TA, Bibb WF, Nokkaew C, Wells DE, Arnow PM, Woods TC, Plikaytis BD: Antigenic and genetic variation in Legionella pneumophila serogroup 6. J Clin Microbiol 1989;27:738-742.
13 Edelstein PH: Improved semiselective medium for isolation of Legionella pneumophila from contaminated clinical and environmental specimens. J Clin Microbiol 1981;14: 298-303.

14 Mustafa AS, Ahmed A, Abal AT, Chugh TD: Establishment and evaluation of a multiplex polymerase chain reaction for detection of mycobacteria and specific identification of Mycobacterium tuberculosis complex. Tuber Lung Dis 1995;76:336-343.

15 Qasem JA, Khan ZU, Mustafa AS: Diagnosis of nocardiosis by polymerase chain reaction: an experimental study in mice. Microbiol Res 2001;156:317-322.

-16 Telenti A, Marchesi F, Balz M, Bally F, Bottger EC, Bodmer T: Rapid identification of mycobacteria to the species level by polymerase chain reaction and restriction enzyme analysis. J Clin Microbiol 1993;31:175178 .

17 Amoudy HA, Al-Turab MB, Mustafa AS: Identification of transcriptionally active open reading frames within the RD1 genomic segment of Mycobacterium tuberculosis. Med Princ Pract 2006;15:137-144.

18 Williams JG, Kubelik AR, Livak KJ, Rafalski JA, Tingey SV: DNA polymorphisms amplified by arbitrary primers are useful as genetic markers. Nucleic Acids Res 1990;18:65316535. 
19 Welsh J, McClelland M: Fingerprinting genomes using PCR with arbitrary primers. Nucleic Acids Res 1990;18:7213-7218.

20 Kumar S, Tamura K, Nei M: MEGA: Molecular Evolutionary Genetics Analysis, Version 1.02. University Park, Pennsylvania State University, 1993.

21 Vaneechoutte M, Van Eldere J: The possibilities and limitations of nucleic acid amplification technology in diagnostic microbiology. J Med Microbiol 1997;46:188-194.

22 Donlan RM: Biofilm formation: a clinically relevant microbiological process. Clin Infect Dis 2001;33:1387-1392.
23 Fliermans CB: Ecology of Legionella: from data to knowledge with a little wisdom. Microb Ecol 1996;32:203-228.

24 Verissimo A, Marrao G, da Silva FG, da Costa MS: Distribution of Legionella spp in hydrothermal areas in continental Portugal and the island of Sao Miguel, Azores. Appl Environ Microbiol 1991;57:2921-2927.

25 Luck PC, Leupold I, Hlawitschka M, et al: Prevalence of Legionella species, serogroups and monoclonal subgroups in hot water systems in south-eastern Germany. Zentralbl Hyg Umweltmed 1993;193:450-460.

-26 Zeitz B, Wiese F, Brengelmann F, Dunkelberg H: Presence of Legionellaceae in warm water supplies and typing of stains by polymerase chain reaction. Epidemiol Infect 2001;126:147-152.
27 Ta AC, Stout JE, Yu Vl, Wagener MM: Comparison of culture methods for monitoring Legionella species in hospital potable water systems and recommendation for standardization of such methods. J Clin Microbiol 1995;33:2118-2123.

28 Ieven M, Goossens H: Relevance of nucleic acid amplification techniques for diagnosis of respiratory tract infections in the clinical laboratory. Clin Microbiol Rev 1997; 10:242256.

29 Ruef C: Nosocomial Legionnaires' disease Strategies for prevention. J Microbiol Methods 1998;33:81-91. 